E R R A T U M

Corrigendum to Volume 12, Number 1, pp. 22-26

Published online: 28 January 2010

(CFESEO 2010

\title{
Contribution of hypoxia-measuring molecular imaging techniques to radiotherapy planning and treatment
}

\author{
Carlos Ferrer Albiach • Antonio Conde Moreno - Marta Rodríguez Cordón • \\ Virginia Morillo Macías • Ana Bouché Babiloni • Inmaculada Beato Tortajada • \\ Ángel Sánchez Iglesias • Alicia Francés Muñoz
}

Unfortunately, in the table 1 there was a mistake.

The correct Table 1 is:

Table 1 Comparison of radiotracers and hypoxia-detection methods

\begin{tabular}{lllllll}
\hline Techniques & Invasiveness & $\begin{array}{l}\text { Requires } \\
\text { injection }\end{array}$ & Measures & $\begin{array}{l}\text { Clinical validation } \\
\text { in RT }\end{array}$ & $\begin{array}{l}\text { General availability } \\
(1-5)\end{array}$ & $\begin{array}{l}\text { Monitors changes } \\
\text { in } \mathrm{pO}_{2}\end{array}$ \\
\hline$\left[{ }^{18} \mathrm{~F}\right]$ FMISO PET & No & Yes & Hypoxia & \pm & 3 & No \\
{$\left[{ }^{18}\right.$ F]FAZA PET } & No & Yes & Hypoxia & No & 2 & No \\
{$\left[{ }^{18}\right.$ F]FETNIM PET } & No & Yes & Hypoxia & No & 1 & No \\
{$\left[{ }^{18}\right.$ F]EF5 PET } & No & Yes & Hypoxia & \pm & 2 & No \\
{$\left[{ }^{64} \mathrm{Cu}\right]$ ATSM/PET } & No & Yes & Hypoxia & \pm & 3 & No \\
IA-IAZGP & No & Yes & Hypoxia & No & 1 & No \\
BOLD MRI & No & Yes & DHb IN RBCs & Yes & 4 & Yes \\
Polarographic method & Yes & & PO & Yes & 2 & No \\
Immunohistochemical & Yes & No & Hypoxia & Yes & 4 & \\
\hline
\end{tabular}

Modified from Padhani A, et al. Eur Radiol 2007(17):861-872

The original version of the article can be found in:

Clin Transl Oncol (2010) 12:22-26

DOI 10.1007/s12094-010-0462-3 